\title{
Analysis of nucleotide-binding oligomerization domain proteins in a murine model of pneumococcal meningitis
}

Xinjie Liu ${ }^{1,2^{*}}$, Qizheng $\operatorname{Han}^{3}$ and Junhong Leng ${ }^{4}$

\begin{abstract}
Background: The innate immune system recognizes pathogens via its pattern recognition receptors. The objective of this study was to investigate the role of the nucleotide-binding oligomerization domain (NOD) proteins, a family of the novel bacterial pattern recognition receptors, in host responses to the gram-positive bacteria Streptococcus pneumoniae.
\end{abstract}

Methods: Sprague-Dawley rats were infected via intracisternal injections of viable S. pneumoniae, and rats in the control group were injected with sterile saline. After infection, real-time PCR was performed to determine the presence of mRNAs encoding NOD1 and NOD2. Quantitative analyses of the NOD1, NOD2 and NF-kB proteins were also performed western blotting following challenge infections with viable S. pneumoniae. The TNF-a and IL-6 levels in brain homogenates were evaluated using enzyme-linked immunosorbent assays (ELISAs).

Results: The results revealed up-regulations of the mRNA and protein levels of NOD2 within the CNS of rats with S. pneumoniae meningitis. Moreover, the activation of NF-KB in the brain tissues following infection with live $S$. pneumoniae was also significantly increased, which indicates that NOD2 mediated NF-KB activation in experimental pneumococcal meningitis. Similarly, TNF-a and IL-6 levels were increased in the brain following in vivo S. pneumoniae administration.

Conclusions: These results suggest that NOD2 is involved in the host response to the gram-positive bacteria S. pneumoniae in the CNS and that NOD2 might play an important role in the initiation and/or progression of CNS inflammation associated with pneumococcal meningitis.

Keywords: Meningitis, Gram-positive bacteria, NOD2, Cytokines

\section{Background}

Bacterial meningitis is a serious and often intractable condition that affects the meninges and the brain parenchyma. Streptococcus pneumoniae is a common cause of bacterial meningitis, and such infections are associated with neurological sequelae and a high mortality rate [1-3]. Once Streptococcus pneumoniae have gained access to the central nervous system, the presence of multiplying bacteria within the subarachnoid and ventricular spaces triggers an intense inflammatory host response [4-7].

\footnotetext{
* Correspondence: liuxinjie@sdu.edu.cn

'Department of Pediatrics, Qilu Hospital, Shandong University, No. 107 Wen Hua Xi Road, Jinan, People's Republic of China

${ }^{2}$ Brain Science Research Institute, Shandong University, No. 107 Wen Hua Xi Road, Jinan, People's Republic of China

Full list of author information is available at the end of the article
}

A compelling body of evidence showing that members of the nucleotide-binding oligomerization domain (NOD) family of proteins play a critical role in the immune response following pathogen invasion has accumulated, and at least two members of this family of proteins appear to sense the cytoplasmic microbial pathogen-associated molecular patterns (PAMPs) of the invading microbes and to initiate the activation of the innate immune system, which leads to inflammation [8-10]. NOD1, also called caspase recruitment domain (CARD)-4 protein, appears to interact with motifs found in the peptidoglycans of gram-negative bacteria $[11,12]$. In contrast, it has been suggested that NOD2 (CARD15) recognizes the bacterial molecules that are produced during the synthesis and/ or degradation of peptidoglycan (PGN) [13] and acts a 
general sensor for most bacteria $[7,8]$. Recent studies have demonstrated that microglia and astrocytes express members of the NOD family of proteins that recognize conserved bacterial motifs. To date, the expressions of these novel bacterial pattern recognition receptors have not been established within the central nervous system (CNS) in vivo.

In the present study, we provide a demonstration that murine brains constitutively express low levels of NOD2 and show that this expression is up-regulated following the in vivo administration of Streptococcus pneumoniae. The notion that NOD2 is functional within brains is supported by the observation that this gram-positive CNS pathogen simultaneously elicits increases in the levels of NF-kB protein, which is an essential downstream effector molecule of NOD2-mediated immune responses. Finally, further compelling evidence for the functionality of NOD2 expression in the CNS is provided by a demonstration of the augmentation of inflammatory cytokine production. Taken together, these findings demonstrate that the expression of functional NOD2 proteins in murine brains represents a potentially important mechanism of bacterial infections of the CNS.

\section{Methods}

\section{Animals and experimental design}

This study was performed in strict accordance with the guidelines of the NIH's Guide for the Care and Use of Laboratory Animals (National Institutes of Health Publication No. 80-23, revised 1996). The protocol was approved by the Animal Care Committee of Shandong University. A total of 48 2-week-old Sprague-Dawley rats were obtained from the Model Animal Research Center of Shandong University (Jinan, PR China). The rats were randomly divided into three infected groups (D1, D2 and D3) and one control group (12 rats in each group). The rats in the infected groups were inoculated with $S$. pneumoniae and sacrificed on day 1 (D1), D2, and D3 after inoculation. The control group was inoculated with sterile saline and sacrificed at $24 \mathrm{~h}$ after injection.

\section{Infecting organism}

The serotype 3 strain of $S$. pneumoniae (provided by the National Institute for the Control of Pharmaceutical and Biological Products, Beijing, China) is one of the most common causes of neonatal meningitis and was used in this study. The bacteria was grown on sheep blood agar plates, cultured overnight in VITAL AER broth and incubated overnight at $37^{\circ} \mathrm{C}$ in air with $5 \% \mathrm{CO}_{2}$ as described previously by our laboratory. The culture broth was centrifuged, pelleted, resuspended in sterile saline to the desired density and used for intracisternal injection. The inoculum was routinely checked for purity and density with quantitative cultures.

\section{Intracisternal administration of the bacteria}

The operation to induce bacterial meningitis has been previously described by our laboratory [7]. Briefly, infection was induced by direct intracisternal injection of $10 \mu \mathrm{l}$ of saline containing $1 \times 10^{6}$ of viable S. pneumoniae via a 32-gauge needle. Uninfected animals were injected with $10 \mu \mathrm{l}$ of sterile saline. To confirm the development of bacterial meningitis, CSF was obtained by puncture of the cisterna magna $24 \mathrm{~h}$ after inoculation and then cultured, and the brains of the rats were histologically examined. At 1,2 and 3 days postinfection, the animals were killed, and all brain tissues were removed. The brain tissue was prepared for assessments of NOD1 and NOD2 mRNAs by reverse transcription-PCR (RT-PCR), analyses of the NOD1, NOD2 and NF-kB proteins by western blotting and exploration of the cytokines with specific capture ELISA.

\section{Reverse transcription-PCR (RT-PCR)}

Total RNA from brain tissue was extracted using Trizol reagent following the manufacturer's instructions. mRNA was isolated by using the Trizol kit and converted to cDNA using the reverse transcriptase. The positive and negative strand PCR primers that were used to amplify the mRNA encoding murine NOD1 (299 bp fragment) were, respectively [13] GTCCTCAACGAGCATGGCGA GACT and AGCTCATCCAGGCCGTCAA. The positive and negative strand primers used to amplify the mRNA encoding murine NOD2 (273 bp fragment) were GCT GCCAATCTTCACGTCGTC and TAAGTACTGAGGA AGCGAGACTGA, respectively. The primers were synthesized by Shanghai Sangon Biological Engineering.

Technology Company Limited. The NOD1 and NOD2 mRNA levels were measured via comparisons to the mRNA level of the housekeeping gene $\beta$-actin that were made with a PCR-based method for the quantitation of mRNA that has been described previously in detail by our laboratory [7].

\section{Western blot analyses of the NOD1, NOD2 and NF-kB protein levels}

The quantitative analyses of the NOD1, NOD2 and NF$\mathrm{kB}$ protein levels were accomplished with western blot analyses that were performed essentially as previously described [13]. The rats were killed at the indicated times after infection, and all brain tissue was removed. Protein extraction performed using a Total Protein Extraction Kit and a Nuclear-Cytosol Extraction Kit. The total NOD1 and NOD2 proteins and nuclear NF- $\mathrm{kB}$ proteins were prepared. Protein content was measured with a BCA kit according to manufacturer's instructions. The total protein samples (50 ug) and nuclear protein samples (30 ug) were subjected to electrophoresis on $10 \%$ sodium dodecyl sulfate-polyacrylamide gels (SDS/PAGE) and transferred to nitrocellulose membranes. The membranes were blocked 
with $5 \%$ skimmed milk/TPBS (10 mM Tris- $\mathrm{HCl}, 150 \mathrm{mM}$ $\mathrm{NaCl}, 0.05 \%$ Tween-20) for $2 \mathrm{~h}$ at room temperature and then incubated with anti-NOD1 (1:500, Cell Signaling Technology), anti-NOD2 (1: 500, Cell Signaling Technology) and anti-NF-kB P65 antibody (1:500, Santa Cruz Biotechnology). The membranes were washed and incubated with secondary antibody for $1 \mathrm{~h}$, and the relative densities of the bands were then analyzed.

\section{Quantification of cytokine secretion}

The TNF- $\alpha$ and IL-6 levels in the brain homogenates were determined using a commercially available ELISA kit according to the directions provided by the manufacturer (Santa Cruz Biotechnology). The cytokine levels in the brain homogenates were normalized to total brain weight and are reported as ng/g of brain tissue.

\section{Statistical analyses}

The data are presented as the means \pm the S.D.s, and oneway ANOVA analyses were performed with SPSS13.0 software to compare the differences. A value of $\mathrm{p}<0.05$ was considered statistically significant.

\section{Results}

\section{S. pneumoniae-induced NOD2 mRNA expression in the} brain tissue

To determine whether the murine brain tissue expressed these novel pattern recognition receptors, we assessed the NOD1 and NOD2 mRNA levels in the uninfected and S. pneumoniae-infected murine brains. At 1, 2 and 3 days postinfection, RNA was isolated, and real-time
PCR was performed to determine the presence of the NOD1 and NOD2 mRNAs. As shown in Figure 1, NOD1 and NOD2 mRNAs were constitutively expressed at very low levels across the entire brains of the uninfected rats. S. pneumoniae administration failed to elicit significant increases in NOD1 expression (Figure 1A,B). The $S$. pneumoniae-infected animals exhibited a significant elevation in NOD2 mRNA (Figure 1C,D).

\section{S. pneumoniae-mediated increases in NOD2 protein in the} brain tissue following in vivo bacterial administration

To determine whether the expression of NOD mRNAs in the brain tissue translated into the expression of the NOD proteins, we performed western blot analyses. As shown in Figure 2, the murine brain tissue constitutively expressed low levels of NOD1 and NOD2 proteins or failed to express these proteins at all. Importantly, S. pneumoniae administration resulted in marked increases in NOD2 protein expression. In contrast, the brains expressed little NOD1 protein following bacterial challenge.

\section{Increased NF-kB expression during acute CNS inflamma- tion following S. pneumoniae administration}

To determine whether the NOD2 protein is functional in rats, we investigated whether the murine brain tissue expressed NF-kB, which is a critical downstream effector molecule in NOD2-mediated cellular activation. As shown in Figure 3, the brains of the uninfected animals expressed robust levels of this protein. However, the brains of the $S$. pneumoniae-infected rats exhibit significantly higher levels of NF-kB protein expression (Figure 3).
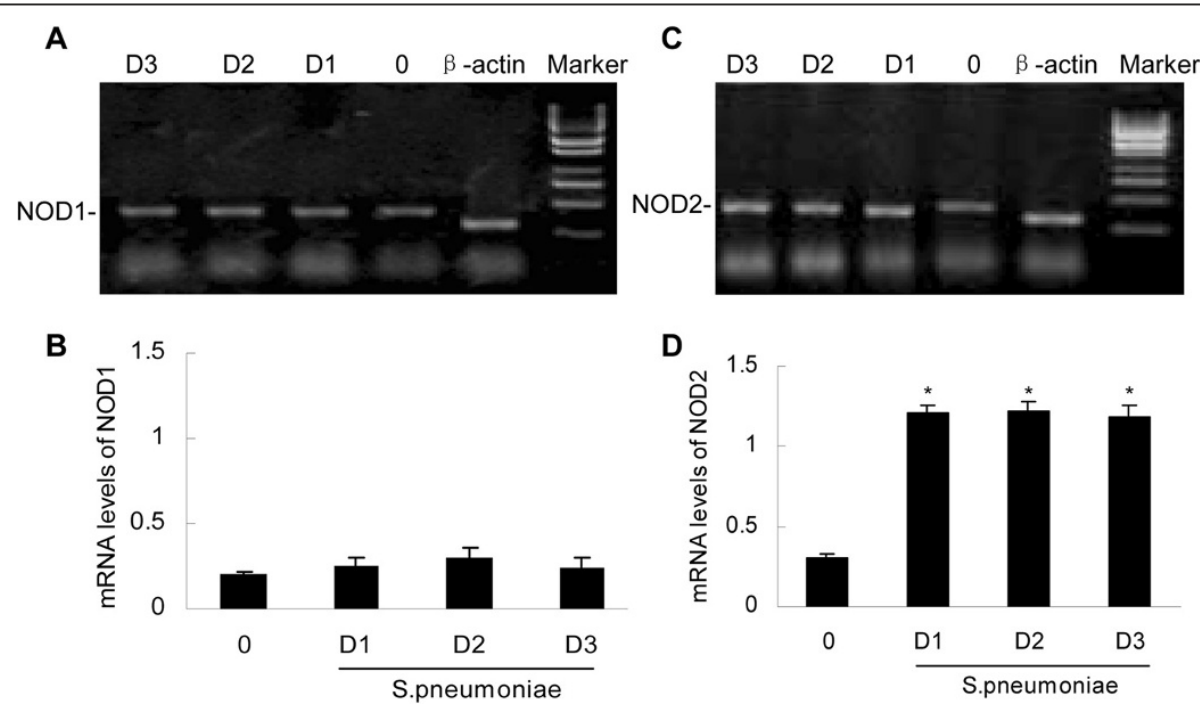

D

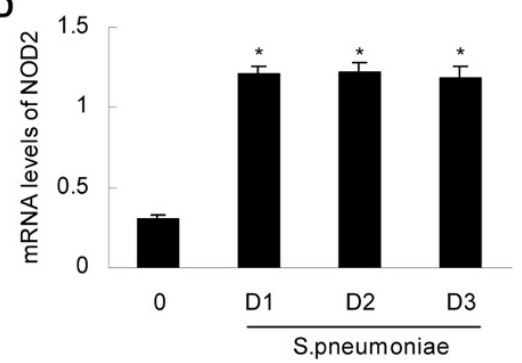

Figure 1 Electrophoretic determination of the expressions of NOD1 and NOD2 mRNAs (A and C). Vehicle (0) or S. pneumoniae $\left(1 \times 10^{6}\right.$ bacteria) was administered to the rats via intracisternal injection. At days 1 (D1), D2, and D3 postinfection, brain tissue homogenates were isolated for measurements of NOD1 (Panel $\mathbf{A}$ ) and NOD2 (Panel $\mathbf{C}$ ). The expressions of NOD1 and NOD2 mRNAs were quantified by densitometric analyses and normalized to $\beta$-actin expression (Panels $\mathbf{B}$ and $\mathbf{D}$ ). The asterisks indicate statistically significant differences compared to the corresponding uninfected animals $(p<0.05)$. 

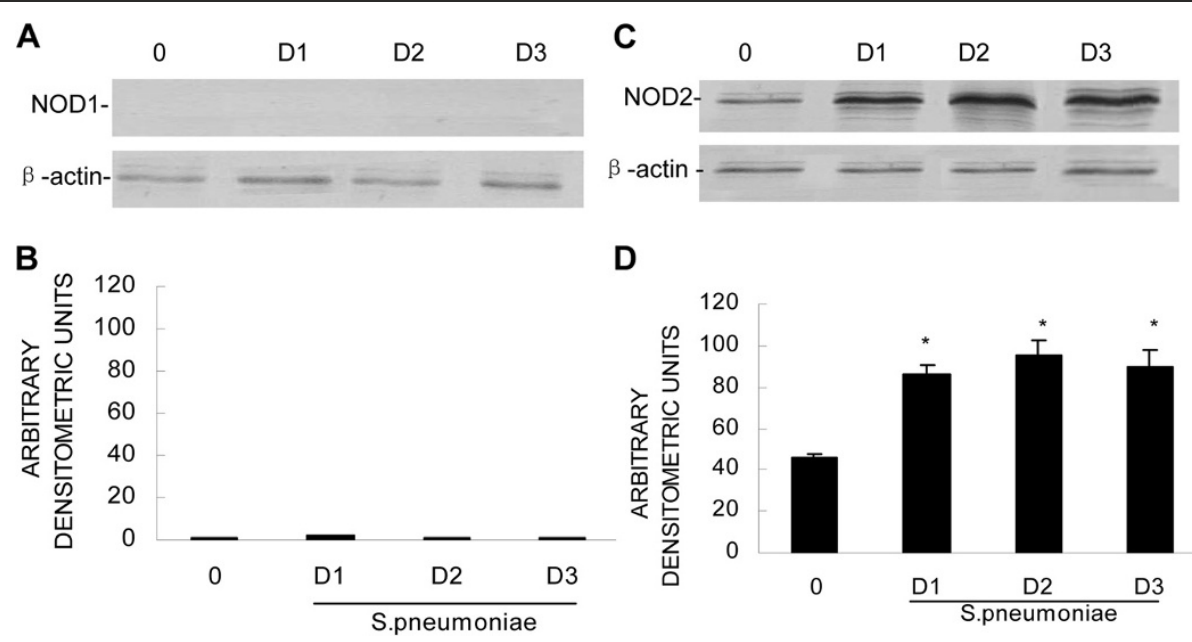

Figure 2 Western blot analyses of the NOD1 and NOD2 proteins (A and C). The rats were administered vehicle (0) or were infected with S. pneumoniae $\left(1 \times 10^{6}\right.$ bacteria) via intracisternal injection. At days 1 (D1), D2, and D3 postinfection, protein isolates were assayed for the presence of NOD1 (Panel $\mathbf{A}$ ) and NOD2 (Panel $\mathbf{C}$ ). NOD1 and NOD2 protein levels were quantified by densitometric analyses and normalized to $\beta$-actin expression (Panels $\mathbf{B}$ and $\mathbf{D}$ ). The asterisks indicate statistically significant differences compared to the corresponding uninfected animals $(p<0.05)$.

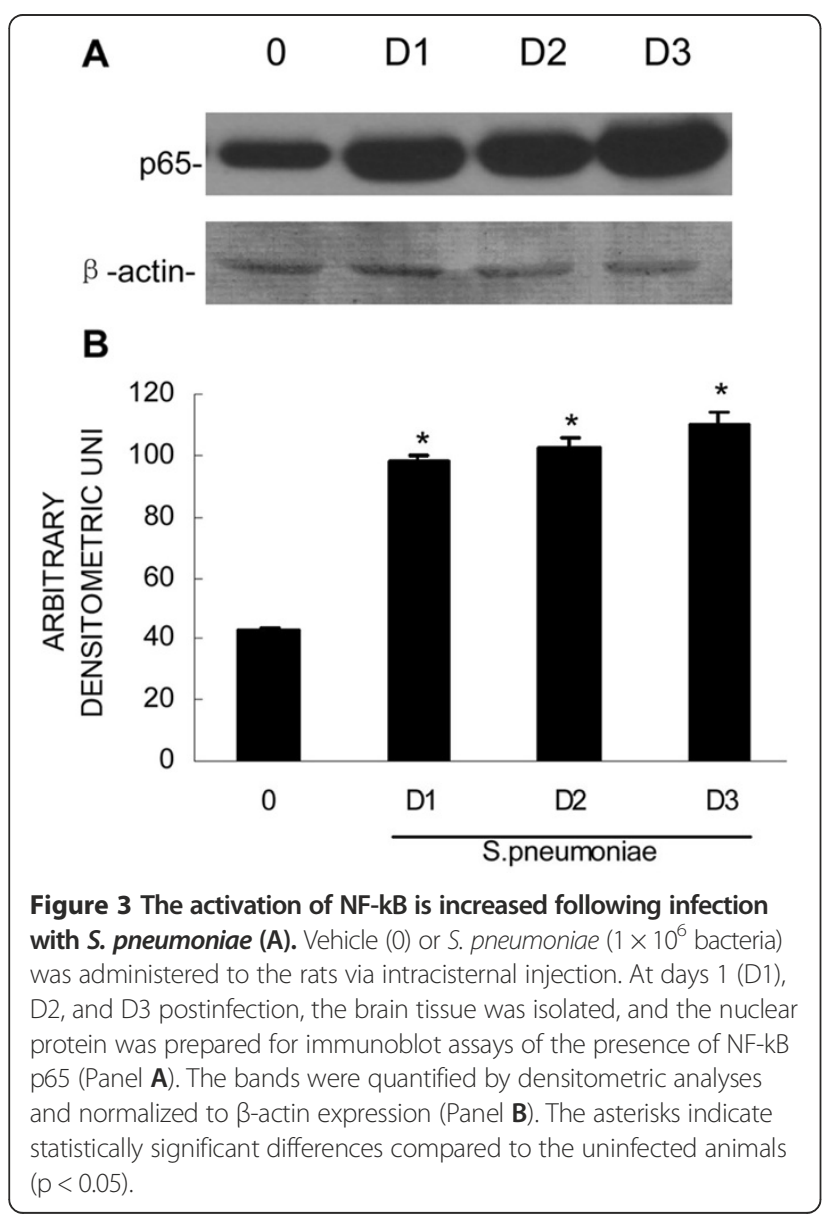

\section{S. pneumoniae-associated increases in inflammatory cytokine levels within the CNS}

To further evaluate the role of NOD2 protein expression in S. pneumoniae-induced neuroinflammation, we assessed the levels of the key inflammatory mediators within the CNS. As shown in Figure 4, S. pneumoniae administration significantly increased the levels of the inflammatory cytokines TNF- $\alpha$ (Figure $4 \mathrm{~A}$ ) and IL-6(Figure 4B) in the CNS.

\section{Discussion}

The innate immune system recognizes pathogens via its pattern recognition receptors. In pneumococcal meningitis, multiple microbial pattern recognition receptors including Toll-like family of the receptors (TLRs) were expressed to perceive bacterial pathogens and to initiate potentially damaging CNS inflammation Klein et al. [14] have recently demonstrated that TLR2 and TLR4 expressed on radioresistant and transplanted BM-derived cells were major cellular sensors of invading S. pneumoniae inducing inflammatory responses in murine S. pneumoniae meningitis. Mogensen et al. [15] also demonstrated that live $S$. pneumoniae activate the inflammatory response through Toll-like receptors 2, 4, and 9 in species-specific patterns. However, the expression of these receptors does not preclude the involvement of other pattern recognition receptors in the perception of bacterial pathogens. Another subset of pattern recognition receptors are the recently identified nucleotide-binding oligomerization domain (NOD) proteins.

In the present study, we assessed the expression levels of NOD mRNAs and proteins during host recognition of $S$. pneumoniae. We provided evidence for the up-regulations of the expressions of the mRNAs and proteins of these 

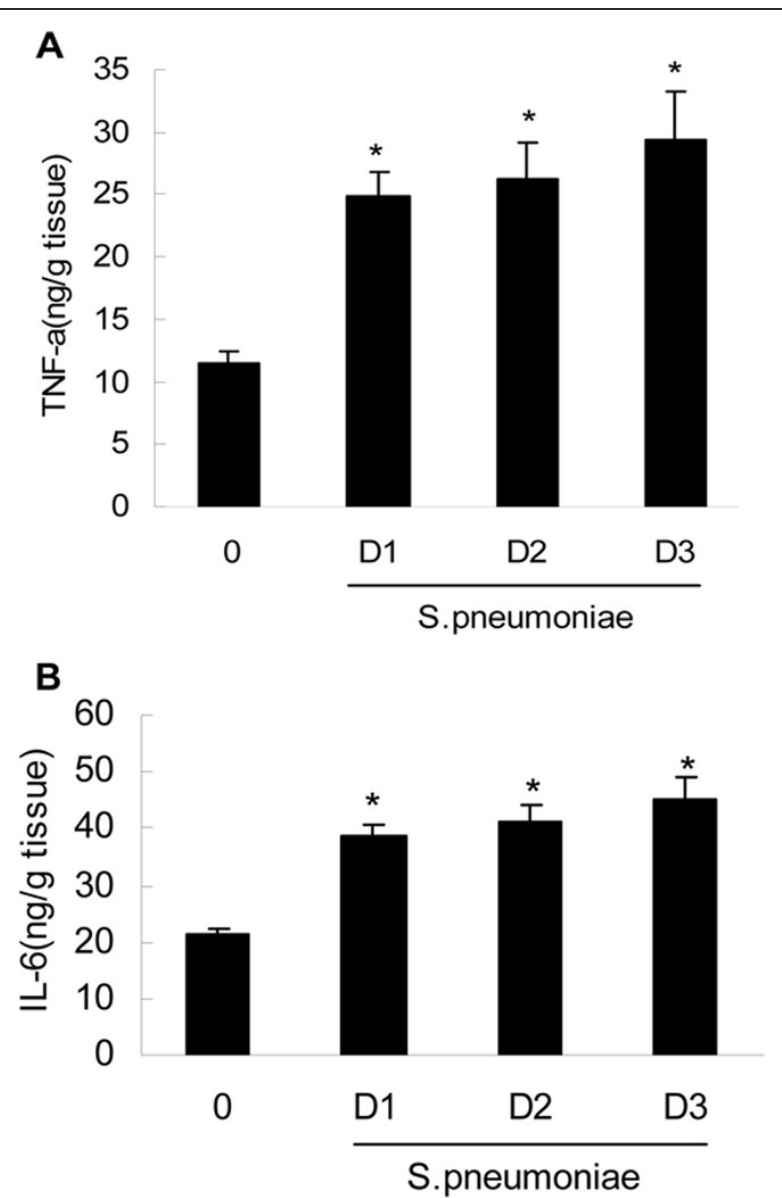

Figure 4 S. pneumoniae administration elicited elevations in inflammatory cytokine levels within the CNS (A and B). Infection was induced via direct intracisternal injection of vehicle (0) or $S$. pneumoniae $\left(1 \times 10^{6}\right.$ bacteria). On day 1 (D1), D2, and D3 postinfection, brain tissue homogenates were isolated for assessment of TNF-a (Panel A) and IL-6 (Panel B) protein expression by specific capture ELISA. The asterisks indicate statistically significant differences compared to the uninfected animals $(p<0.05)$.

novel pathogen-associated molecular pattern receptors within the CNS following the in vivo administration of $S$. pneumoniae. We demonstrated that rat brain tissue constitutively expresses low levels of NOD2 mRNA and detectable levels of NOD2 protein. The expression of NOD2 mRNA and protein were markedly elevated following infection with the gram-positive pathogen S. pneumoniae. These observations are consistent with the previously documented ability of murine glial cells to express NOD2 $[13,16,17]$, which suggests the involvement of NOD2 proteins in the immune response to S. pneumoniae in brain tissue [18]. Currently, the precise mechanisms responsible for this phenomenon have not been determined, although it is possible that the increase in NOD2 expression might result from the recruitment of immune system cells that express NOD2 and not necessarily from an up-regulation of NOD2 expression in the brain cells.
In contrast to our findings related to NOD2, our data indicated that uninfected brain tissues express little or no NOD1, and only a modest level of expression was detectable following infection with the gram-positive bacteria $S$. pneumoniae. This finding agrees with the work of others that has demonstrated that microglia express very low levels of NOD1 mRNA and that exposure of microglia to viable Neisseria meningitis fails to elicit significant increases in NOD1 expression [13]. While the reasons for these findings remain unclear, it is possible that NOD1 only interacts with the naturally occurring peptidoglycan degradation product GlcNAc-MurNAC-LAla-gamma-D-Glu-meso-DAP that is found in the peptidoglycans of gram-negative bacteria $[11,12]$. More interesting, others [9] have described that intestinal epithelial cells express NOD1 almost exclusively and that gram-negative bacteria are the leading cause of intestinal infections. In contrast, NOD2 appears to function as an intracellular receptor for a minimal motif that is common to all bacterial peptidoglycans $[19,20]$.

In the present study, we showed that the activation of the pivotal inflammatory transcriptional activator NF-kB in brain tissues following infection with live $S$. pneumoniae was significantly increased. NF-kB binding sites have been identified in the NOD2 promoter [21,22], and NOD2 has been reported to activate the pivotal transcription factor NF-kB. NOD2 recruits the adaptor molecules RICK (also called RIP2) and CARD9, and the activations of these molecules eventually lead to NF-kB activation [23,24]. Thus, the present demonstrations of NOD2 expression and inducible NF-kB activation in the brain tissue following the in vivo administration of $S$. pneumoniae lend credence to the notion that NOD2 mediates NF-kB activation during host responses to this gram-positive CNS pathogen [3]. Thus, NOD2 might be functional in the host CNS.

A growing body of evidence suggests that once bacteria have gained access to the central nervous system, the pathogens initiate CNS inflammation and that inflammatory mediators are released in this process. Accordingly, others and we have demonstrated the ability of murine glial cells to produce key inflammatory cytokines, including TNF- $\alpha$ and IL-6, in response to bacterial pathogens [25-27]. In the present study, we measured the productions of key inflammatory cytokines following S. pneumoniae infection in vivo. We showed that the infection of rats with $S$. pneumoniae elicited significant increases in inflammatory cytokine production.

Importantly, we observed a simultaneous occurrence of rapid increases in inflammatory mediators, an elevation in NOD2 protein and an increase in NF-k B activation following in vivo S. pneumoniae administration. Together, these finding suggest a physiological role of the NOD2 protein in the immune response to pneumococci in the brain tissue. 


\section{Conclusions}

Based on the observations of the present study, we demonstrated that NOD2 is involved in host responses to gram-positive bacteria $S$. pneumoniae in the CNS and that NOD2 plays an important role in the initiation and/ or progression of the CNS inflammation that is associated with pneumococcal meningitis. Nevertheless, the role of NOD2 in the host response to the gram-positive bacteria Streptococcus pneumoniae is currently controversial. NF-kB activation can induce NOD2 up-regulation, and it remains unclear whether NOD2 is activated or plays a role in the inflammatory process that is triggered by $S$. pneumonia. Therefore, further studies are needed to further elucidate these issues. Knowledge of the molecular interactions of pneumococci with brain tissue might lead to improvements in disease outcome.

\section{Competing interests}

The authors declare that they have no competing interests.

\section{Authors' contributions}

Conceived and designed the experiments: XL. Performed the experiments: $\mathrm{XL}$. Analyzed the data: $\mathrm{QH}$. Contributed analysis tools: $\mathrm{QH}$. Wrote the paper: $\mathrm{XL}$. JL contributed to the revision of the manuscript. All authors read and approved the final manuscript.

\section{Acknowledgments}

This research was supported by the Science and Technology Project Plan of the Department of Science \& Technology of Shandong Province (2010GSF10262), by the Independent Innovation Project of (201004057) the Jinan City Science and Technology Bureau, by the National Natural Science Foundation of China (81371337), and by the Special Foundation for Taishan Scholars.

\section{Author details}

${ }^{1}$ Department of Pediatrics, Qilu Hospital, Shandong University, No. 107 Wen Hua Xi Road, Jinan, People's Republic of China. ${ }^{2}$ Brain Science Research Institute, Shandong University, No. 107 Wen Hua Xi Road, Jinan, People's Republic of China. ${ }^{3}$ Department of Respiratory Medicine, Provincial Hospital Affiliated to Shandong University, No. 4 Duan Xing Xi Road, Jinan, People's Republic of China. ${ }^{4}$ Department of Ultrasonic Diagnosis, Jinan Maternity and Children Care Hospital, Jian Guo Xiao Jing San Road, Jinan, People's Republic of China.

Received: 1 November 2013 Accepted: 21 November 2014

Published online: 02 December 2014

\section{References}

1. Meli DN, Christen S, Leib SL, Täuber MG: Current concepts in the pathogenesis of meningitis caused by Streptococcus pneumoniae. Curr Opin Infect Dis 2002, 15:253-257.

2. Scheld WM, Koedel U, Nathan B, Pfister HW: Pathophysiology of bacterial meningitis: mechanism(s) of neuronal injury. J Infect Dis 2002, 186:S225-S233.

3. Opitz B, Püschel A, Schmeck B, Hocke AC, Rosseau S: Nucleotide-binding oligomerization domain proteins are innate immune receptors for internalized Streptococcus pneumoniae. J Biol Chem 2004, 279:36426-36432.

4. Meli DN, Coimbra RS, Erhart DG, Loquet G, Bellac CL: Doxycycline reduces mortality and injury to the brain and cochlea in experimental pneumococcal meningitis. Infect Immun 2006, 74:3890-3896.

5. Chauhan VS, Kluttz JM, Bost KL, Marriott I: Prophylactic and therapeutic targeting of the neurokinin-1 receptor limits neuroinflammation in a murine. model of pneumococcal meningitis. J Immunol 2011, 186:7255-7263.

6. Leib SL, Clements JM, Lindberg RLP, Heimgartner C, Loeffler JM: Inhibition of matrix metalloproteinases and tumour necrosis factor alpha converting enzyme as adjuvant therapy in pneumococcal meningitis. Brain 2001, 124:1734-1742.
7. Xinjie L, Qizheng H, Ruopeng S, Zhaoxia L: Dexamethasone regulation of matrix metalloproteinase expression in experimental pneumococcal meningitis. Brain Res 2008, 1207:237-243.

8. Girardin SE, Sansonetti PJ, Philpott PJ: Intracellular vs extracellular recognition of pathogens-common concepts in mammals and flies. Trends Microbiol 2002, 10:193-199.

9. Strober W, Murray PJ, Kitani A, Watanable T: Signalling pathways and molecular interactions of NOD1 and NOD2. Nat Rev Immunol 2006, 6:9-29.

10. Inohara, Chamaillard, McDonald C, Nuñez G: NOD-LRR proteins: role in host-microbial interactions and inflammatory disease. Annu Rev Biochem 2005, 74:355-383.

11. Chamaillard M, Hashimoto M, Horie Y, Masumoto J, Qiu S: An essential role for NOD1 in host recognition of bacterial peptidoglycan containing diaminopimelic acid. Nat Immunol 2003, 4:702-707.

12. Girardin SE, Boneca IG, Cameiro LA, Antignac A, Jehanno M: NOD1 detects a unique muropeptide from gram-negative bacterial peptidoglycan. Science 2003, 300:1584-1587.

13. Sterka D Jr, Marriott I: Characterization of nucleotide-binding oligomerization domain (NOD) protein expression in primary murine microglia. J Neuroimmunol 2006, 179:65-75.

14. Klein M, Obermaier B, Angele B, Pfister HW, Wagner H, Koedel U, Kirschning CJ: Innate immunity to pneumococcal infection of the central nervous system depends on toll-like receptor (TLR) 2 and TLR4. J Infect Dis 2008, 198:1028-1036.

15. Mogensen TH, Paludan SR, Kilian M, Ostergaard L: Live Streptococcus pneumoniae, Haemophilus influenzae, and Neisseria meningitidis activate the inflammatory response through Toll-like receptors 2, 4, and 9 in species-specific patterns. J Leukoc Biol 2006, 80:267-277.

16. Chauhan VS, Sterka DG Jr, Furr SR, Young AB, Marriott I: NOD2 plays an important role in the inflammatory responses of microglia and astrocytes to bacterial CNS pathogens. Glia 2009, 57:414-423.

17. Sterka D Jr, Rati DM, Marriott I: Functional expression of NOD2, a novel pattern recognition receptor for bacterial motifs, in primary murine satrocytes. Glia 2006, 53:322-330.

18. Liu X, Chauhan VS, Young AB, Marriott I: NOD2 mediates inflammatory responses of primary murine glia to Streptococcus pneumoniae. Glia 2010, 58:839-847.

19. Fournier B, Philpott DJ: Recognition of Staphylococcus aureus by the innate immune system. Clin Microbiol Rev 2005, 18:521-540.

20. Takada H, Uehara A: Enhancement of TLR-mediated innate immune responses by peptidoglycans through NOD signaling. Curr Pharm Des 2006, 12:4163-4172.

21. Gutierrez O, Pipaon C, Inohara N, Fontalba A, Ogura Y: Induction of Nod2 in myelomonocytic and intestinal epithelial cells via nuclear factor-kappa B activation. J Biol Chem 2002, 277:41701-41705

22. Rosenstiel P, Fantini M, Brautigam K, Kuhbacher T, Waetzig GH: TNF-alpha and IFN-gamma regulate the expression of the NOD2 (CARD15) gene in human intestinal epithelial cells. Gastroenterology 2003, 124:1001-1009.

23. Hsu YM, Zhang Y, You Y, Wang D, Li H: The adaptor protein CARD9 is required for innate immune responses to intracellular pathogens. Nat Immunol 2007, 8:198-205.

24. Kobayashi K, Inohara N, Hernandez LD, Galan JE, Nunez G: RICK/Rip2/ CARDIAK mediate signaling for receptors of the innate and adaptive immune systems. Nature 2002, 416:194-199.

25. Liu X, Chauhan VS, Marriott I: NOD2 contributes to the inflammatory responses of primary murine microglia and astrocytes to Staphylococcus aureus. Neurosci Lett 2010, 474:93-98.

26. Carpentier PA, Begolka WS, Olson JK, Elhofy A, Karpus WJ: Differential activation of astrocytes by innate and adaptive immune stimuli. Glia 2005, 49:360-374.

27. Esen N, Kielian T: Central role for MyD88 in the responses of microglia to athogen-associated molecular patterns. J Immunol 2006, 176:6802-6811.

doi:10.1186/s12879-014-0648-3

Cite this article as: Liu et al:: Analysis of nucleotide-binding oligomerization domain proteins in a murine model of pneumococcal meningitis. BMC Infectious Diseases 2014 14:648. 